\title{
New subclass of the class of close-to-convex harmonic mappings defined by a third-order differential inequality
}

\author{
Serkan Çakmak*(D), Elif Yaşar (D), Sibel Yalçın (D) \\ Department of Mathematics, Faculty of Arts and Sciences, \\ Bursa Uludă̆ University, 16059, Görükle, Bursa, Turkey
}

\begin{abstract}
In this paper, we introduce a new subclass of harmonic functions $\mathfrak{f}=\mathfrak{s}+\overline{\mathfrak{t}}$ in the open unit disk $\mathcal{U}=\{z \in \mathbb{C}:|z|<1\}$ satisfying

$\operatorname{Re}\left[\gamma \mathfrak{s}^{\prime}(z)+\delta z \mathfrak{s}^{\prime \prime}(z)+\left(\frac{\delta-\gamma}{2}\right) z^{2} \mathfrak{s}^{\prime \prime \prime}(z)-\lambda\right]>\left|\gamma \mathfrak{t}^{\prime}(z)+\delta z \mathfrak{t}^{\prime \prime}(z)+\left(\frac{\delta-\gamma}{2}\right) z^{2} \mathfrak{t}^{\prime \prime \prime}(z)\right|$, where $0 \leq \lambda<\gamma \leq \delta, z \in \mathcal{U}$. We determine several properties of this class such as close-toconvexity, coefficient bounds, and growth estimates. We also prove that this class is closed under convex combination and convolution of its members. Furthermore, we investigate the properties of fully starlikeness and fully convexity of the class.
\end{abstract}

Mathematics Subject Classification (2020). 30C45, 30C50

Keywords. harmonic, univalent, close-to-convex, coefficient estimates, convolution

\section{Introduction}

Let $\mathcal{H}$ denote the class of complex-valued harmonic functions $\mathfrak{f}=\mathfrak{s}+\overline{\mathfrak{t}}$ defined in the open unit disk $\mathcal{U}=\{z \in \mathbb{C}:|z|<1\}$, and normalized by $\mathfrak{f}(0)=\mathfrak{f}_{z}(0)-1=0$. Also, let $\mathcal{H}^{0}=\left\{\mathfrak{f} \in \mathcal{H}: \mathfrak{f}_{\bar{z}}(0)=0\right\}$. Each function $\mathfrak{f} \in \mathcal{H}^{0}$ can be expressed as $\mathfrak{f}=\mathfrak{s}+\overline{\mathfrak{t}}$, where

$$
\mathfrak{s}(z)=z+\sum_{m=2}^{\infty} a_{m} z^{m}, \quad \mathfrak{t}(z)=\sum_{m=2}^{\infty} b_{m} z^{m}
$$

are analytic in $\mathcal{U}$. A necessary and sufficient condition for $\mathfrak{f}$ to be locally univalent and sense-preserving in $\mathcal{U}$ is that $\left|\mathfrak{s}^{\prime}(z)\right|>\left|\mathfrak{t}^{\prime}(z)\right|$ in $\mathcal{U}$. See $[5,8]$.

Denote by $\mathcal{S}_{H}$ the class of functions $\mathfrak{f}=\mathfrak{s}+\overline{\mathfrak{t}}$ that are harmonic, univalent and sensepreserving in the unit disk $\mathcal{U}$. Further, let $\mathcal{S}_{H}^{0}=\left\{\mathfrak{f} \in \mathcal{S}_{H}: \mathfrak{f}_{\bar{z}}(0)=0\right\}$. Note that, with $\mathfrak{t}(z)=0$, the classical family $\mathcal{S}$ of analytic univalent and normalized functions in $\mathcal{U}$ is a subclass of $\mathcal{S}_{H}^{0}$, just as the family $\mathcal{A}$ of analytic and normalized functions in $\mathcal{U}$ is a subclass of $\mathcal{H}^{0}$. A simply connected subdomain of $\mathbb{C}$ is said to be close-to-convex if its complement in $\mathbb{C}$ can be written as the union of non-crossing half-lines.

\footnotetext{
*Corresponding Author.

Email addresses: serkan.cakmak64@gmail.com (S. Çakmak), elifyasar@yahoo.com (E. Yaşar), syalcin@uludag.edu.tr (S. Yalçın)

Received: 20.04.2021; Accepted: 06.09.2021
} 
Let $\mathcal{K}, \mathcal{S}^{*}$ and $\mathcal{C}$ be the subclasses of $\mathcal{S}$ mapping $\mathcal{U}$ onto convex, starlike and close-toconvex domains, respectively, just as $\mathcal{K}_{H}^{0}, \mathcal{S}_{H}^{*, 0}$ and $\mathcal{C}_{H}^{0}$ are the subclasses of $\mathcal{S}_{H}^{0}$ mapping $\mathcal{U}$ onto their respective domains.

In [13], Hernandez and Martin introduced the notion of stable harmonic mappings. A sense-preserving harmonic mapping $\mathfrak{f}=\mathfrak{s}+\overline{\mathfrak{t}}$ is said to be stable harmonic univalent (resp. stable harmonic convex, stable harmonic starlike, or stable harmonic close-to-convex) in $\mathcal{U}$, if all functions $\mathfrak{f}_{\epsilon}=\mathfrak{s}+\epsilon \overline{\mathfrak{t}}$ with $|\epsilon|=1$ are univalent (resp. convex, starlike, or closeto-convex) in $\mathcal{U}$. It is proved that $\mathfrak{f}=\mathfrak{s}+\bar{t}$ is stable harmonic univalent (resp. convex, starlike, or close-to-convex) if and only if $F_{\epsilon}=\mathfrak{s}+\epsilon \mathfrak{t}$ are univalent (resp. convex, starlike, or close-to-convex) in $\mathcal{U}$ for each $|\epsilon|=1$.

Recall that, convexity and starlikeness are hereditary properties for conformal mappings and they do not extend to harmonic functions [8]. The failure of hereditary properties leads to the notion of fully starlike and fully convex functions which introduced by Chuaqui, Duren and Osgood [4]. A harmonic function $\mathfrak{f}$ of the unit disk is said to be fully convex, if it maps every circle $|z|=r<1$ in a one-to-one manner onto a convex curve. Such a harmonic mapping $\mathfrak{f}$ with $\mathfrak{f}(0)=0$ is fully starlike if it maps every circle $|z|=r<1$ in a one-to-one manner onto a curve that bounds a domain starlike with respect to the origin. Denote by $\mathcal{F K}_{H}^{0}$ and $\mathcal{F} \mathcal{S}_{H}^{*, 0}$ the subclasses of $\mathcal{K}_{H}^{0}$ and $\mathcal{S}_{H}^{*, 0}$ consisting of fully convex and fully starlike functions, respectively. In 2013, Nagpal and Ravichandran [16] introduced the concept of fully starlike functions of order $\alpha(0 \leq \alpha<1)$ and fully convex functions of order $\alpha$ for certain families of univalent harmonic mappings. In 2019, Ghosh and Vasudevarao [11] considered the particular case of generalized Bernardi integral operator of harmonic functions that satisfy the conditions of the harmonic Bieberbach coefficient conjecture and obtained the radius of fully starlikeness and the radius of fully convexity of that harmonic operator. In $[13,16,17]$, it is proved that stable harmonic convex (or stable harmonic starlike) mappings in $\mathcal{U}$ are fully convex (or fully starlike) in $\mathcal{U}$.

In 2014, Nagpal and Ravichandran [17] studied a class $W_{H}^{0}$ of functions $\mathfrak{f} \in \mathcal{H}^{0}$ satisfying the condition $\operatorname{Re}\left[\mathfrak{s}^{\prime}(z)+z \mathfrak{s}^{\prime \prime}(z)\right]>\left|\mathfrak{t}^{\prime}(z)+z \mathfrak{t}^{\prime \prime}(z)\right|$ for $z \in \mathcal{U}$ which is harmonic analogue of the class $W$ defined by Chichra [3] consisting of functions $\mathfrak{f} \in \mathcal{A}$ satisfying the condition $\operatorname{Re}\left[\mathfrak{f}^{\prime}(z)+z \mathfrak{f}^{\prime \prime}(z)\right]>0$ for $z \in \mathcal{U}$. It is stated that $W_{H}^{0} \subset \mathcal{S}_{H}^{*, 0}$ and in particular, the members of the class are fully starlike in $\mathcal{U}$.

Ghosh and Vasudevarao [10] investigated radius of convexity for the partial sums of members of the class $W_{H}^{0}(\delta)$ of functions $\mathfrak{f} \in \mathcal{H}^{0}$ satisfying the condition $\operatorname{Re}\left[\mathfrak{s}^{\prime}(z)+\delta z \mathfrak{s}^{\prime \prime}(z)\right]>\left|\mathfrak{t}^{\prime}(z)+\delta z \mathfrak{t}^{\prime \prime}(z)\right|$ for $\delta \geq 0$, and $z \in \mathcal{U}$.

Further, Rajbala and Prajapat [18] studied the class $W_{H}^{0}(\delta, \lambda)$ of functions $\mathfrak{f} \in \mathcal{H}^{0}$ satisfying the condition $\operatorname{Re}\left[\mathfrak{s}^{\prime}(z)+\delta z \mathfrak{s}^{\prime \prime}(z)-\lambda\right]>\left|\mathfrak{t}^{\prime}(z)+\delta z \mathfrak{t}^{\prime \prime}(z)\right|$ for $\delta \geq 0,0 \leq \lambda<1$, and $z \in \mathcal{U}$. They constructed harmonic polynomials involving Gaussian hypergeometric function which belong to the class $W_{H}^{0}(\delta, \lambda)$.

Very recently, Yaşar and Yalçın [23] introduced the class $R_{H}^{0}(\delta, \gamma)$ of functions $\mathfrak{f} \in \mathcal{H}^{0}$ satisfying the condition

$$
\operatorname{Re}\left[\mathfrak{s}^{\prime}(z)+\delta z \mathfrak{s}^{\prime \prime}(z)+\gamma z^{2} \mathfrak{s}^{\prime \prime \prime}(z)\right]>\left|\mathfrak{t}^{\prime}(z)+\delta z \mathfrak{t}^{\prime \prime}(z)+\gamma z^{2} \mathfrak{t}^{\prime \prime \prime}(z)\right|
$$

for $\delta \geq \gamma \geq 0, z \in \mathcal{U}$.

In all studies mentioned above $[10,17,18,23]$, it is proved that the functions in corresponding classes are close-to-convex. Also, coefficient bounds, growth estimates, and convolution properties of the classes are obtained.

Denote by $\mathcal{R}_{H}^{0}(\gamma, \delta, \lambda)$, the class of functions $\mathfrak{f}=\mathfrak{s}+\overline{\mathfrak{t}} \in \mathcal{H}^{0}$ and satisfy

$$
\operatorname{Re}\left[\gamma \mathfrak{s}^{\prime}(z)+\delta z \mathfrak{s}^{\prime \prime}(z)+\left(\frac{\delta-\gamma}{2}\right) z^{2} \mathfrak{s}^{\prime \prime \prime}(z)-\lambda\right]>\left|\gamma \mathfrak{t}^{\prime}(z)+\delta z \mathfrak{t}^{\prime \prime}(z)+\left(\frac{\delta-\gamma}{2}\right) z^{2} \mathfrak{t}^{\prime \prime \prime}(z)\right|
$$


where $0 \leq \lambda<\gamma \leq \delta$.

It is evident that $W_{H}^{0} \equiv W_{H}^{0}(1) \equiv \mathcal{R}_{H}^{0}(1,1,0), W_{H}^{0}(1, \lambda) \equiv \mathcal{R}_{H}^{0}(1,1, \lambda), R_{H}^{0}\left(\delta, \frac{\delta-1}{2}\right) \equiv$ $\mathcal{R}_{H}^{0}(1, \delta, 0)$.

Let $\mathcal{R}(\gamma, \delta, \lambda)$ denote a class of functions $\mathfrak{f} \in \mathcal{A}$ such that

$$
\operatorname{Re}\left\{\gamma \mathfrak{f}^{\prime}(z)+\delta z f^{\prime \prime}(z)+\left(\frac{\delta-\gamma}{2}\right) z^{2} \mathfrak{f}^{\prime \prime \prime}(z)\right\}>\lambda \quad(0 \leq \lambda<\gamma \leq \delta) .
$$

The class $\mathcal{R}(\gamma, \delta, \lambda)$ is a particular case of the class which is studied by Al-Refai [19]. The starlikeness and convexity of the class $\mathcal{R}(1, \delta, \lambda)$ are studied in $[2,20]$.

In this paper, we mainly deal with the functions $\mathfrak{f}=\mathfrak{s}+\overline{\mathfrak{t}} \in \mathcal{H}^{0}$ of the class $\mathcal{R}_{H}^{0}(\gamma, \delta, \lambda)$ which is defined by the third-order differential inequality (1.2). In the second section, we prove that the members of the class $\mathcal{R}_{H}^{0}(\gamma, \delta, \lambda)$ are close-to-convex. We also obtain coefficient bounds, growth estimates, and sufficient coefficient condition of this class. In the third section, we prove that this class is closed under convex combination and convolution of its members. In the last section, we investigate the radii of fully starlikeness and fully convexity of the class $\mathcal{R}_{H}^{0}(\gamma, \delta, \lambda)$, and we give a result due to the class $\mathcal{R}_{H}^{0}(1, \delta, \lambda)$ using previous works [2] and [17].

\section{Close-to-convexity, coefficient bounds, growth estimates}

First, we give a result of Clunie and Sheil-Small [5] which derives a sufficient condition for $\mathfrak{f} \in \mathcal{H}$ to be close-to-convex.

Lemma 2.1. Suppose $\mathfrak{s}$ and $\mathfrak{t}$ are analytic in $\mathfrak{U}$ with $\left|\mathfrak{t}^{\prime}(0)\right|<\left|\mathfrak{s}^{\prime}(0)\right|$ and $F_{\epsilon}=\mathfrak{s}+\epsilon \mathfrak{t}$ is close-to-convex for each $\epsilon(|\epsilon|=1)$, then $\mathfrak{f}=\mathfrak{s}+\overline{\mathfrak{t}}$ is close-to-convex in $\mathcal{U}$.

Theorem 2.2. The harmonic mapping $\mathfrak{f}=\mathfrak{s}+\overline{\mathfrak{t}} \in \mathcal{R}_{H}^{0}(\gamma, \delta, \lambda)$ if and only if $F_{\epsilon}=\mathfrak{s}+\epsilon \mathfrak{t} \in$ $\mathcal{R}(\gamma, \delta, \lambda)$ for each $\epsilon(|\epsilon|=1)$.

Proof. Suppose $\mathfrak{f}=\mathfrak{s}+\overline{\mathfrak{t}} \in \mathcal{R}_{H}^{0}(\gamma, \delta, \lambda)$. For each $|\epsilon|=1$,

$$
\begin{aligned}
& \operatorname{Re}\left\{\gamma F_{\epsilon}^{\prime}(z)+\delta z F_{\epsilon}^{\prime \prime}(z)+\left(\frac{\delta-\gamma}{2}\right) z^{2} F_{\epsilon}^{\prime \prime \prime}(z)\right\} \\
& =\operatorname{Re}\left\{\gamma \mathfrak{s}^{\prime}(z)+\delta z \mathfrak{s}^{\prime \prime}(z)+\left(\frac{\delta-\gamma}{2}\right) z^{2} \mathfrak{s}^{\prime \prime \prime}(z)\right. \\
& \left.+\epsilon\left(\gamma \mathfrak{t}^{\prime}(z)+\delta z \mathfrak{t}^{\prime \prime}(z)+\left(\frac{\delta-\gamma}{2}\right) z^{2} \mathfrak{t}^{\prime \prime \prime}(z)\right)\right\} \\
& >\operatorname{Re}\left\{\gamma \mathfrak{s}^{\prime}(z)+\delta z \mathfrak{s}^{\prime \prime}(z)+\left(\frac{\delta-\gamma}{2}\right) z^{2} \mathfrak{s}^{\prime \prime \prime}(z)\right\} \\
& -\left|\gamma \mathfrak{t}^{\prime}(z)+\delta z \mathfrak{t}^{\prime \prime}(z)+\left(\frac{\delta-\gamma}{2}\right) z^{2} \mathfrak{t}^{\prime \prime \prime}(z)\right| \\
& >\lambda \quad(z \in \mathcal{U}) .
\end{aligned}
$$

Thus, $F_{\epsilon} \in \mathcal{R}(\gamma, \delta, \lambda)$ for each $\epsilon(|\epsilon|=1)$. Conversely, let $F_{\epsilon}=\mathfrak{s}+\epsilon \mathfrak{t} \in \mathcal{R}(\gamma, \delta, \lambda)$ then

$$
\begin{aligned}
& \operatorname{Re}\left\{\gamma \mathfrak{s}^{\prime}(z)+\delta z \mathfrak{s}^{\prime \prime}(z)+\left(\frac{\delta-\gamma}{2}\right) z^{2} \mathfrak{s}^{\prime \prime \prime}(z)\right\} \\
> & \operatorname{Re}\left\{-\epsilon\left(\gamma \mathfrak{t}^{\prime}(z)+\delta z \mathfrak{t}^{\prime \prime}(z)+\left(\frac{\delta-\gamma}{2}\right) z^{2} \mathfrak{t}^{\prime \prime \prime}(z)\right)\right\}+\lambda(z \in \mathcal{U}) .
\end{aligned}
$$

With appropriate choice of $\epsilon(|\epsilon|=1)$, it follows that

$$
\begin{array}{r}
\operatorname{Re}\left\{\gamma \mathfrak{s}^{\prime}(z)+\delta z \mathfrak{s}^{\prime \prime}(z)+\left(\frac{\delta-\gamma}{2}\right) z^{2} \mathfrak{s}^{\prime \prime \prime}(z)-\lambda\right\} \\
>\left|\gamma \mathfrak{t}^{\prime}(z)+\delta z \mathfrak{t}^{\prime \prime}(z)+\left(\frac{\delta-\gamma}{2}\right) z^{2} \mathfrak{t}^{\prime \prime \prime}(z)\right|(z \in \mathcal{U}),
\end{array}
$$


and hence $\mathfrak{f} \in \mathcal{R}_{H}^{0}(\gamma, \delta, \lambda)$.

Lemma 2.3. (Jack-Miller-Mocanu Lemma [14,15]) Let $w$ defined by $w(z)=c_{n} z^{n}+$ $c_{n+1} z^{n+1}+\ldots$ be analytic in $\mathcal{U}$, with $c_{n} \neq 0$, and let $z_{0} \neq 0, z_{0}=r_{0} e^{i \theta_{0}}\left(0<r_{0}<1\right)$ be a point of $\mathcal{U}$ such that

$$
\left|w\left(z_{0}\right)\right|=\max _{|z| \leq\left|z_{0}\right|}|w(z)|
$$

then there is a real number $k, k \geq n \geq 1$, such that

$$
\frac{z_{0} w^{\prime}\left(z_{0}\right)}{w\left(z_{0}\right)}=k \text { and } \operatorname{Re}\left\{1+\frac{z_{0} w^{\prime \prime}\left(z_{0}\right)}{w^{\prime}\left(z_{0}\right)}\right\} \geq k
$$

Lemma 2.4. If $F \in \mathcal{R}(\gamma, \delta, \lambda)$ then $\operatorname{Re}\left\{F^{\prime}(z)\right\}>0$, and hence $F$ is close-to-convex in $\mathcal{U}$.

Proof. Suppose $F \in \mathcal{R}(\gamma, \delta, \lambda)$ and $\frac{2 \gamma F^{\prime}(z)+2 \delta z F^{\prime \prime}(z)+(\delta-\gamma) z^{2} F^{\prime \prime \prime}(z)-2 \lambda}{2(\gamma-\lambda)}=: \Psi(z)$. Then $\operatorname{Re}\{\Psi(z)\}>0$ for $z \in \mathcal{U}$. Consider an analytic function $w$ in $\mathcal{U}$ with $w(0)=0$ and

$$
F^{\prime}(z)=\frac{1+w(z)}{1-w(z)}, \quad w(z) \neq 1
$$

We need to prove that $|w(z)|<1$ for all $z \in \mathcal{U}$. Then we have

$$
\begin{aligned}
\Psi(z)= & \frac{2 \gamma F^{\prime}(z)+2 \delta z F^{\prime \prime}(z)+(\delta-\gamma) z^{2} F^{\prime \prime \prime}(z)-2 \lambda}{2(\gamma-\lambda)} \\
= & \frac{\gamma}{\gamma-\lambda} \frac{1+w(z)}{1-w(z)}+\frac{2 \delta}{\gamma-\lambda} \frac{z w^{\prime}(z)}{(1-w(z))^{2}} \\
& +\frac{\delta-\gamma}{\gamma-\lambda} \frac{z^{2}\left[w^{\prime \prime}(z)(1-w(z))+2\left(w^{\prime}(z)\right)^{2}\right]}{(1-w(z))^{3}}-\frac{\lambda}{\gamma-\lambda} \\
= & \frac{1}{\gamma-\lambda}\left(\gamma \frac{1+w(z)}{1-w(z)}+2 \delta \frac{z w^{\prime}(z)}{(1-w(z))^{2}}\right. \\
& \left.+(\delta-\gamma) \frac{z w^{\prime}(z)}{(1-w(z))^{2}} \frac{z w^{\prime \prime}(z)}{w^{\prime}(z)}+2(\delta-\gamma) \frac{\left(z w^{\prime}(z)\right)^{2}}{(1-w(z))^{3}}-\lambda\right)
\end{aligned}
$$

Since $w$ is analytic in $\mathcal{U}$ and $w(0)=0$, if there is $z_{0} \in \mathcal{U}$ such that

$$
\max _{|z| \leq\left|z_{0}\right|}|w(z)|=\left|w\left(z_{0}\right)\right|=1
$$

then by Lemma 2.3 , we can write

$$
w\left(z_{0}\right)=e^{i \theta}, \quad z_{0} w^{\prime}\left(z_{0}\right)=k w\left(z_{0}\right)=k e^{i \theta}, \quad(k \geq 1,0<\theta<2 \pi) .
$$

and

$$
\operatorname{Re}\left\{\frac{z_{0} w^{\prime \prime}\left(z_{0}\right)}{w^{\prime}\left(z_{0}\right)}\right\} \geq k-1
$$


For such a point $z_{0} \in \mathcal{U}$, we obtain

$$
\begin{aligned}
\operatorname{Re}\left\{\Psi\left(z_{0}\right)\right\}= & \frac{1}{\gamma-\lambda} \operatorname{Re}\left(\gamma \frac{1+w\left(z_{0}\right)}{1-w\left(z_{0}\right)}+2 \delta \frac{z_{0} w^{\prime}\left(z_{0}\right)}{\left(1-w\left(z_{0}\right)\right)^{2}}\right. \\
& \left.+(\delta-\gamma) \frac{z_{0} w^{\prime}\left(z_{0}\right)}{\left(1-w\left(z_{0}\right)\right)^{2}} \frac{z_{0} w^{\prime \prime}\left(z_{0}\right)}{w^{\prime}\left(z_{0}\right)}+2(\delta-\gamma) \frac{\left(z_{0} w^{\prime}\left(z_{0}\right)\right)^{2}}{\left(1-w\left(z_{0}\right)\right)^{3}}-\lambda\right) \\
= & \frac{1}{\gamma-\lambda}\left[-\frac{\delta k}{1-\cos \theta}-\frac{(\delta-\gamma) k}{2(1-\cos \theta)} \operatorname{Re}\left\{\frac{z w^{\prime \prime}\left(z_{0}\right)}{w^{\prime}\left(z_{0}\right)}\right\}+\frac{(\delta-\gamma) k^{2}}{2(1-\cos \theta)}-\lambda\right] \\
\leq & \frac{1}{\gamma-\lambda}\left[-\frac{\delta k}{1-\cos \theta}+\frac{(\delta-\gamma) k}{2(1-\cos \theta)}(1-k)+\frac{(\delta-\gamma) k^{2}}{2(1-\cos \theta)}-\lambda\right] \\
= & -\frac{1}{\gamma-\lambda}\left[\frac{(\delta+\gamma) k}{2(1-\cos \theta)}+\lambda\right]<0,
\end{aligned}
$$

which contradicts our assumption. Hence, there is no $z_{0} \in \mathcal{U}$ such that $\left|w\left(z_{0}\right)\right|=1$, which means that $|w(z)|<1$ for all $z \in \mathcal{U}$. Therefore, we obtain that $\operatorname{Re}\left\{F^{\prime}(z)\right\}>0$.

Theorem 2.5. The functions in the class $\mathcal{R}_{H}^{0}(\gamma, \delta, \lambda)$ are close-to-convex in $\mathcal{U}$.

Proof. Referring to Lemma 2.4, we derive that functions $F_{\epsilon}=\mathfrak{s}+\epsilon \mathfrak{t} \in \mathcal{R}(\gamma, \delta, \lambda)$ are close-to-convex in $\mathcal{U}$ for each $\epsilon(|\epsilon|=1)$. Now in view of Lemma 2.1 and Theorem 2.2, we obtain that functions in $\mathcal{R}_{H}^{0}(\gamma, \delta, \lambda)$ are close-to-convex in $\mathcal{U}$.

Theorem 2.6. Let $\mathfrak{f}=\mathfrak{s}+\overline{\mathfrak{t}} \in \mathcal{R}_{H}^{0}(\gamma, \delta, \lambda)$ then for $m \geq 2$,

$$
\left|b_{m}\right| \leq \frac{2(\gamma-\lambda)}{m^{2}[2 \gamma+(\delta-\gamma)(m-1)]}
$$

The result is sharp and equality holds for the function $\mathfrak{f}(z)=z+\frac{2(\gamma-\lambda)}{m^{2}[2 \gamma+(\delta-\gamma)(m-1)]} \bar{z}^{m}$.

Proof. Suppose that $\mathfrak{f}=\mathfrak{s}+\overline{\mathfrak{t}} \in \mathcal{R}_{H}^{0}(\gamma, \delta, \lambda)$. Using the series representation of $\mathfrak{t}(z)$, we derive

$$
\begin{aligned}
& r^{m-1} m^{2}\left[\gamma+\frac{\delta-\gamma}{2}(m-1)\right]\left|b_{m}\right| \\
\leq & \frac{1}{2 \pi} \int_{0}^{2 \pi}\left|\gamma \mathfrak{t}^{\prime}\left(r e^{i \theta}\right)+\delta r e^{i \theta} \mathfrak{t}^{\prime \prime}\left(r e^{i \theta}\right)+\left(\frac{\delta-\gamma}{2}\right) r^{2} e^{2 i \theta} \mathfrak{t}^{\prime \prime \prime}\left(r e^{i \theta}\right)\right| d \theta \\
< & \frac{1}{2 \pi} \int_{0}^{2 \pi} \operatorname{Re}\left\{\gamma \mathfrak{s}^{\prime}\left(r e^{i \theta}\right)+\delta r e^{i \theta} \mathfrak{s}^{\prime \prime}\left(r e^{i \theta}\right)+\left(\frac{\delta-\gamma}{2}\right) r^{2} e^{2 i \theta} \mathfrak{s}^{\prime \prime \prime}\left(r e^{i \theta}\right)-\lambda\right\} d \theta \\
= & \frac{1}{2 \pi} \int_{0}^{2 \pi} \operatorname{Re}\left\{\gamma-\lambda+\sum_{m=2}^{\infty} m^{2}\left[\gamma+\frac{\delta-\gamma}{2}(m-1)\right] a_{m} r^{m-1} e^{i(m-1) \theta}\right\} d \theta \\
= & \gamma-\lambda .
\end{aligned}
$$

Allowing $r \rightarrow 1^{-}$gives the desired bound. Moreover, it is easy to verify that the equality holds for the function $\mathfrak{f}(z)=z+\frac{2(\gamma-\lambda)}{m^{2}[2 \gamma+(\delta-\gamma)(m-1)]} \bar{z}^{m}$. 
Theorem 2.7. Let $\mathfrak{f}=\mathfrak{s}+\overline{\mathfrak{t}} \in \mathcal{R}_{H}^{0}(\gamma, \delta, \lambda)$. Then for $m \geq 2$, we have

$$
\begin{aligned}
& \text { (i) }\left|a_{m}\right|+\left|b_{m}\right| \leq \frac{4(\gamma-\lambda)}{m^{2}[2 \gamma+(\delta-\gamma)(m-1)]}, \\
& \text { (ii) }|| a_{m}|-| b_{m}|| \leq \frac{4(\gamma-\lambda)}{m^{2}[2 \gamma+(\delta-\gamma)(m-1)]} \\
& \text { (iii) }\left|a_{m}\right| \leq \frac{4(\gamma-\lambda)}{m^{2}[2 \gamma+(\delta-\gamma)(m-1)]}
\end{aligned}
$$

All these results are sharp and all equalities hold for the function

$$
\mathfrak{f}(z)=z+\sum_{m=2}^{\infty} \frac{4(\gamma-\lambda)}{m^{2}[2 \gamma+(\delta-\gamma)(m-1)]} z^{m}
$$

Proof. (i) Suppose that $\mathfrak{f}=\mathfrak{s}+\overline{\mathfrak{t}} \in \mathcal{R}_{H}^{0}(\gamma, \delta, \lambda)$, then from Theorem 2.2, $F_{\epsilon}=\mathfrak{s}+\epsilon \mathfrak{t} \in$ $\mathcal{R}(\gamma, \delta, \lambda)$ for $\epsilon(|\epsilon|=1)$. Thus for each $|\epsilon|=1$, we have

$$
\operatorname{Re}\left\{\gamma(\mathfrak{s}+\epsilon \mathfrak{t})^{\prime}+\delta z(\mathfrak{s}+\epsilon \mathfrak{t})^{\prime \prime}+\left(\frac{\delta-\gamma}{2}\right) z^{2}(\mathfrak{s}+\epsilon \mathfrak{t})^{\prime \prime \prime}\right\}>\lambda
$$

for $z \in \mathcal{U}$. This implies that there exists an analytic function $p$ of the form $p(z)=1+$ $\sum_{m=1}^{\infty} p_{m} z^{m}$, with $\operatorname{Re}[p(z)]>0$ in $\mathcal{U}$ such that

$$
\begin{aligned}
& \gamma \mathfrak{s}^{\prime}(z)+\delta z \mathfrak{s}^{\prime \prime}(z)+\left(\frac{\delta-\gamma}{2}\right) z^{2} \mathfrak{s}^{\prime \prime \prime}(z)+\epsilon\left(\gamma \mathfrak{t}^{\prime}(z)+\delta z \mathfrak{t}^{\prime \prime}(z)+\left(\frac{\delta-\gamma}{2}\right) z^{2} \mathfrak{t}^{\prime \prime \prime}(z)\right) \\
& =\lambda+(\gamma-\lambda) p(z) .
\end{aligned}
$$

Comparing coefficients on both sides of (2.2) we have

$$
m^{2}\left[\gamma+\frac{\delta-\gamma}{2}(m-1)\right]\left(a_{m}+\epsilon b_{m}\right)=(\gamma-\lambda) p_{m-1} \text { for } m \geq 2
$$

Since $\left|p_{m}\right| \leq 2$ for $m \geq 1$, and $\epsilon(|\epsilon|=1)$ is arbitrary, proof of (i) is complete. Proofs of (ii) and (iii) follows from (i). The function $\mathfrak{f}(z)=z+\sum_{m=2}^{\infty} \frac{4(\gamma-\lambda)}{m^{2}[2 \gamma+(\delta-\gamma)(m-1)]} z^{m}$, shows that all inequalities are sharp.

The following result gives a sufficient condition for a function to be in the class $\mathcal{R}_{H}^{0}(\gamma, \delta, \lambda)$.

Theorem 2.8. Let $\mathfrak{f}=\mathfrak{s}+\overline{\mathfrak{t}} \in \mathcal{H}^{0}$ with

$$
\sum_{m=2}^{\infty} m^{2}[2 \gamma+(\delta-\gamma)(m-1)]\left(\left|a_{m}\right|+\left|b_{m}\right|\right) \leq 2(\gamma-\lambda),
$$

then $\mathfrak{f} \in \mathcal{R}_{H}^{0}(\gamma, \delta, \lambda)$.

Proof. Suppose that $\mathfrak{f}=\mathfrak{s}+\overline{\mathfrak{t}} \in \mathcal{H}^{0}$. Then using (2.3), 


$$
\begin{aligned}
& \operatorname{Re}\left\{\gamma \mathfrak{s}^{\prime}(z)+\delta z \mathfrak{s}^{\prime \prime}(z)+\left(\frac{\delta-\gamma}{2}\right) z^{2} \mathfrak{s}^{\prime \prime \prime}(z)-\lambda\right\} \\
= & \operatorname{Re}\left\{\gamma-\lambda+\sum_{m=2}^{\infty} m^{2}\left[\gamma+\frac{\delta-\gamma}{2}(m-1)\right] a_{m} z^{m-1}\right\} \\
> & \gamma-\lambda-\sum_{m=2}^{\infty} m^{2}\left[\gamma+\frac{\delta-\gamma}{2}(m-1)\right]\left|a_{m}\right| \\
\geq & \sum_{m=2}^{\infty} m^{2}\left[\gamma+\frac{\delta-\gamma}{2}(m-1)\right]\left|b_{m}\right| \\
> & \left|\sum_{m=2}^{\infty} m^{2}\left[\gamma+\frac{\delta-\gamma}{2}(m-1)\right] b_{m} z^{m-1}\right| \\
= & \left|\gamma \mathfrak{t}^{\prime}(z)+\delta z \mathfrak{t}^{\prime \prime}(z)+\left(\frac{\delta-\gamma}{2}\right) z^{2} \mathfrak{t}^{\prime \prime \prime}(z)\right| .
\end{aligned}
$$

Hence, $\mathfrak{f} \in \mathcal{R}_{H}^{0}(\gamma, \delta, \lambda)$.

Corollary 2.9. Let $\mathfrak{f}=\mathfrak{s}+\overline{\mathfrak{t}} \in \mathcal{H}^{0}$ satisfies the inequality (2.3), then $f$ is stable harmonic close-to-convex in $\mathrm{U}$.

Theorem 2.10. Let $\mathfrak{f}=\mathfrak{s}+\overline{\mathfrak{t}} \in \mathcal{R}_{H}^{0}(\gamma, \delta, \lambda)$. Then

$$
\begin{aligned}
& |z|+4(\gamma-\lambda) \sum_{m=2}^{\infty} \frac{(-1)^{m-1}|z|^{m}}{m^{2}[2 \gamma+(\delta-\gamma)(m-1)]} \leq|\mathfrak{f}(z)|, \\
& |\mathfrak{f}(z)| \leq|z|+4(\gamma-\lambda) \sum_{m=2}^{\infty} \frac{|z|^{m}}{m^{2}[2 \gamma+(\delta-\gamma)(m-1)]} .
\end{aligned}
$$

Inequalities are sharp for the function $f(z)=z+\sum_{m=2}^{\infty} \frac{4(\gamma-\lambda)}{m^{2}[2 \gamma+(\delta-\gamma)(m-1)]} \bar{z}^{m}$.

Proof. Let $\mathfrak{f}=\mathfrak{s}+\overline{\mathfrak{t}} \in \mathcal{R}_{H}^{0}(\gamma, \delta, \lambda)$. Then using Theorem 2.2, $F_{\epsilon} \in \mathcal{R}(\gamma, \delta, \lambda)$ and for each $|\epsilon|=1$ we have $\operatorname{Re}\{\psi(z)\}>\lambda$ where

$$
\psi(z)=\gamma F_{\epsilon}^{\prime}(z)+\delta z F_{\epsilon}^{\prime \prime}(z)+\frac{\delta-\gamma}{2} z^{2} F_{\epsilon}^{\prime \prime \prime}(z) .
$$

Then, we have

$$
\begin{aligned}
\psi(z) & =\left(\frac{\delta-\gamma}{2}\right)\left[\frac{2 \gamma}{\delta-\gamma} F_{\epsilon}^{\prime}(z)+\left(\frac{2 \gamma}{\delta-\gamma}+2\right) z F_{\epsilon}^{\prime \prime}(z)+z^{2} F_{\epsilon}^{\prime \prime \prime}(z)\right] \\
& =\left(\frac{\delta-\gamma}{2}\right)\left[\frac{2 \gamma}{\delta-\gamma}\left(z F_{\epsilon}^{\prime}(z)\right)^{\prime}+\left(z^{2} F_{\epsilon}^{\prime \prime}(z)\right)^{\prime}\right] \\
& =\left(\frac{\delta-\gamma}{2}\right)\left[\frac{2 \gamma}{\delta-\gamma}\left(z F_{\epsilon}^{\prime}(z)\right)+\left(z^{2} F_{\epsilon}^{\prime \prime}(z)\right)\right]^{\prime} \\
& =\left(\frac{\delta-\gamma}{2}\right)\left[z^{2-\frac{2 \gamma}{\delta-\gamma}}\left(z^{\frac{2 \gamma}{\delta-\gamma}} F_{\epsilon}^{\prime}(z)\right)^{\prime}\right]^{\prime} .
\end{aligned}
$$

Then integrating from 0 to $z$ gives

$$
\left(\frac{2}{\delta-\gamma}\right) z^{\frac{2 \gamma}{\delta-\gamma}-2} \int_{0}^{z} \psi(\omega) d \omega=\left(z^{\frac{2 \gamma}{\delta-\gamma}} F_{\epsilon}^{\prime}(z)\right)^{\prime} .
$$


Making the substitution $\omega=r^{\frac{\delta-\gamma}{2}} z$ in the above integral and integrating again, change of variables gives

$$
F_{\epsilon}^{\prime}(z)=\frac{1}{\gamma} \int_{0}^{1} \int_{0}^{1} \psi\left(v^{\frac{\delta-\gamma}{2 \gamma}} u z\right) d u d v
$$

On the other hand, since $\operatorname{Re}\left\{\frac{\psi(z)-\lambda}{\gamma-\lambda}\right\}>0$ then $\psi(z) \prec \frac{\gamma+(\gamma-2 \lambda) z}{1-z}$ where $\prec$ denotes the subordination [7]. Let

$$
\phi(z)=\int_{0}^{1} \int_{0}^{1} \frac{d u d v}{1-u v^{\frac{\delta-\gamma}{2 \gamma}} z}=1+\sum_{m=1}^{\infty} \frac{z^{m}}{(1+m)\left(1+\frac{\delta-\gamma}{2 \gamma} m\right)}
$$

and

$$
h(z)=\frac{1}{\gamma}\left(\frac{\gamma+(\gamma-2 \lambda) z}{1-z}\right)=1+\sum_{m=1}^{\infty} \frac{2(\gamma-\lambda)}{\gamma} z^{m}
$$

Then, from (2.4) we have

$$
\begin{aligned}
F_{\epsilon}^{\prime}(z) & \prec(\phi * h)(z) \\
& =\left(1+\sum_{m=1}^{\infty} \frac{z^{m}}{(1+m)\left(1+\frac{\delta-\gamma}{2 \gamma} m\right)}\right) *\left(1+\sum_{m=1}^{\infty} \frac{2(\gamma-\lambda)}{\gamma} z^{m}\right) \\
& =1+\sum_{m=1}^{\infty} \frac{4(\gamma-\lambda)}{m^{2}(\delta-\gamma)+m(\delta+\gamma)+2 \gamma} z^{m} .
\end{aligned}
$$

Since

$$
\begin{aligned}
\left|F_{\epsilon}^{\prime}(z)\right| & =\left|\mathfrak{s}^{\prime}(z)+\epsilon \mathfrak{t}^{\prime}(z)\right| \\
& \leq 1+4(\gamma-\lambda) \sum_{m=1}^{\infty} \frac{|z|^{m}}{m^{2}(\delta-\gamma)+m(\delta+\gamma)+2 \gamma}
\end{aligned}
$$

and

$$
\begin{aligned}
\left|F_{\epsilon}^{\prime}(z)\right| & =\left|\mathfrak{s}^{\prime}(z)+\epsilon \mathbf{t}^{\prime}(z)\right| \\
& \geq 1+4(\gamma-\lambda) \sum_{m=1}^{\infty} \frac{(-1)^{m}|z|^{m}}{m^{2}(\delta-\gamma)+m(\delta+\gamma)+2 \gamma}
\end{aligned}
$$

in particular we have

$$
\left|\mathfrak{s}^{\prime}(z)\right|+\left|\mathfrak{t}^{\prime}(z)\right| \leq 1+4(\gamma-\lambda) \sum_{m=1}^{\infty} \frac{|z|^{m}}{m^{2}(\delta-\gamma)+m(\delta+\gamma)+2 \gamma}
$$

and

$$
\left|\mathfrak{s}^{\prime}(z)\right|-\left|\mathfrak{t}^{\prime}(z)\right| \geq 1+4(\gamma-\lambda) \sum_{m=1}^{\infty} \frac{(-1)^{m}|z|^{m}}{m^{2}(\delta-\gamma)+m(\delta+\gamma)+2 \gamma}
$$


Let $\Gamma$ be the radial segment from 0 to $z$, then

$$
\begin{aligned}
|\mathfrak{f}(z)| & =\left|\int_{\Gamma} \frac{\partial \mathfrak{f}}{\partial \zeta} d \zeta+\frac{\partial \mathfrak{f}}{\partial \bar{\zeta}} d \bar{\zeta}\right| \leq \int_{\Gamma}\left(\left|\mathfrak{s}^{\prime}(\zeta)\right|+\left|\mathfrak{t}^{\prime}(\zeta)\right|\right)|d \zeta| \\
& \leq \int_{0}^{|z|}\left(1+4(\gamma-\lambda) \sum_{m=1}^{\infty} \frac{|\tau|^{m}}{m^{2}(\delta-\gamma)+m(\delta+\gamma)+2 \gamma}\right) d \tau \\
& =|z|+4(\gamma-\lambda) \sum_{m=1}^{\infty} \frac{|z|^{m+1}}{(m+1)\left[m^{2}(\delta-\gamma)+m(\delta+\gamma)+2 \gamma\right]} \\
& =|z|+4(\gamma-\lambda) \sum_{m=2}^{\infty} \frac{|z|^{m}}{m\left[(m-1)^{2}(\delta-\gamma)+(m-1)(\delta+\gamma)+2 \gamma\right]} \\
& =|z|+4(\gamma-\lambda) \sum_{m=2}^{\infty} \frac{|z|^{m}}{m^{2}[2 \gamma+(\delta-\gamma)(m-1)]}
\end{aligned}
$$

and

$$
\begin{aligned}
|\mathfrak{f}(z)| & \geq \int_{\Gamma}\left(\left|\mathfrak{s}^{\prime}(\zeta)\right|-\left|\mathfrak{t}^{\prime}(\zeta)\right|\right)|d \zeta| \\
& \geq \int_{0}^{|z|}\left(1+4(\gamma-\lambda) \sum_{m=1}^{\infty} \frac{(-1)^{m}|\tau|^{m}}{m^{2}(\delta-\gamma)+m(\delta+\gamma)+2 \gamma}\right) d \tau \\
& =|z|+4(\gamma-\lambda) \sum_{m=2}^{\infty} \frac{(-1)^{m-1}|z|^{m}}{m^{2}[2 \gamma+(\delta-\gamma)(m-1)]} .
\end{aligned}
$$

\section{Convex combinations and convolutions}

In this section, we prove that the class $\mathcal{R}_{H}^{0}(\gamma, \delta, \lambda)$ is closed under convex combinations and convolutions of its members.

Theorem 3.1. The class $\mathcal{R}_{H}^{0}(\gamma, \delta, \lambda)$ is closed under convex combinations.

Proof. Suppose $\mathfrak{f}_{i}=\mathfrak{s}_{i}+\overline{\mathfrak{t}}_{i} \in \mathcal{R}_{H}^{0}(\gamma, \delta, \lambda)$ for $i=1,2, \ldots, n$ and $\sum_{i=1}^{n} \varrho_{i}=1\left(0 \leq \varrho_{i} \leq 1\right)$. The convex combination of functions $\mathfrak{f}_{i}(i=1,2, \ldots, n)$ may be written as

$$
\mathfrak{f}(z)=\sum_{i=1}^{n} \varrho_{i} \mathfrak{f}_{i}(z)=\mathfrak{s}(z)+\overline{\mathfrak{t}(z)},
$$

where

$$
\mathfrak{s}(z)=\sum_{i=1}^{n} \varrho_{i} \mathfrak{s}_{i}(z) \text { and } \mathfrak{t}(z)=\sum_{i=1}^{n} \varrho_{i} \mathfrak{t}_{i}(z)
$$


Then both $\mathfrak{s}$ and $\mathfrak{t}$ are analytic in $\mathcal{U}$ with $\mathfrak{s}(0)=\mathfrak{t}(0)=\mathfrak{s}^{\prime}(0)-1=\mathfrak{t}^{\prime}(0)=0$ and

$$
\begin{aligned}
& \operatorname{Re}\left\{\gamma \mathfrak{s}^{\prime}(z)+\delta z \mathfrak{s}^{\prime \prime}(z)+\left(\frac{\delta-\gamma}{2}\right) z^{2} \mathfrak{s}^{\prime \prime \prime}(z)-\lambda\right\} \\
= & \operatorname{Re}\left\{\sum_{i=1}^{n} \varrho_{i}\left(\gamma \mathfrak{s}_{i}^{\prime}(z)+\delta z \mathfrak{s}_{i}^{\prime \prime}(z)+\left(\frac{\delta-\gamma}{2}\right) z^{2} \mathfrak{s}_{i}^{\prime \prime \prime}(z)-\lambda\right)\right\} \\
> & \sum_{i=1}^{n} \varrho_{i}\left|\gamma \mathfrak{t}_{i}^{\prime}(z)+\delta z \mathfrak{t}_{i}^{\prime \prime}(z)+\left(\frac{\delta-\gamma}{2}\right) z^{2} \mathfrak{t}_{i}^{\prime \prime \prime}(z)\right| \\
\geq & \left|\gamma \mathfrak{t}^{\prime}(z)+\delta z \mathfrak{t}^{\prime \prime}(z)+\left(\frac{\delta-\gamma}{2}\right) z^{2} \mathfrak{t}^{\prime \prime \prime}(z)\right|
\end{aligned}
$$

showing that $\mathfrak{f} \in \mathcal{R}_{H}^{0}(\gamma, \delta, \lambda)$.

A sequence $\left\{c_{m}\right\}_{m=0}^{\infty}$ of non-negative real numbers is said to be a convex null sequence, if $c_{m} \rightarrow 0$ as $m \rightarrow \infty$, and $c_{0}-c_{1} \geq c_{1}-c_{2} \geq c_{2}-c_{3} \geq \ldots \geq c_{m-1}-c_{m} \geq \ldots \geq 0$. To prove results for convolution, we shall need the following Lemma 3.2 and Lemma 3.3.

Lemma 3.2 ([9]). If $\left\{c_{m}\right\}_{m=0}^{\infty}$ be a convex null sequence, then function

$$
q(z)=\frac{c_{0}}{2}+\sum_{m=1}^{\infty} c_{m} z^{m}
$$

is analytic and $\operatorname{Re}\{q(z)\}>0$ in $\mathcal{U}$.

Lemma 3.3 ([22]). Let the function $p$ be analytic in $\mathcal{U}$ with $p(0)=1$ and $\operatorname{Re}\{p(z)\}>1 / 2$ in $\mathcal{U}$. Then for any analytic function $F$ in $\mathcal{U}$, the function $p * F$ takes values in the convex hull of the image of $\mathcal{U}$ under $F$.

Lemma 3.4. Let $F \in \mathcal{R}(\gamma, \delta, \lambda)$, then $\operatorname{Re}\left\{\frac{F(z)}{z}\right\}>\frac{1}{2}$.

Proof. Suppose $F \in \mathcal{R}(\gamma, \delta, \lambda)$ be given by $F(z)=z+\sum_{m=2}^{\infty} A_{m} z^{m}$, then

$$
\operatorname{Re}\left\{\gamma+\sum_{m=2}^{\infty} m^{2}\left[\gamma+\frac{\delta-\gamma}{2}(m-1)\right] A_{m} z^{m-1}\right\}>\lambda \quad(z \in \mathcal{U})
$$

which is equivalent to $\operatorname{Re}\{p(z)\}>\frac{1}{2}$ in $\mathcal{U}$, where

$$
p(z)=1+\frac{1}{4(\gamma-\lambda)} \sum_{m=2}^{\infty} m^{2}[2 \gamma+(\delta-\gamma)(m-1)] A_{m} z^{m-1} .
$$

Now consider a sequence $\left\{c_{m}\right\}_{m=0}^{\infty}$ defined by

$$
c_{0}=1 \text { and } c_{m-1}=\frac{4(\gamma-\lambda)}{m^{2}[2 \gamma+(\delta-\gamma)(m-1)]} \text { for } m \geq 2 .
$$

It can be easily seen that the sequence $\left\{c_{m}\right\}_{m=0}^{\infty}$ is a convex null sequence. Using Lemma 3.2 , this implies that the function

$$
q(z)=\frac{1}{2}+\sum_{m=2}^{\infty} \frac{4(\gamma-\lambda)}{m^{2}[2 \gamma+(\delta-\gamma)(m-1)]} z^{m-1}
$$

is analytic and $\operatorname{Re}\{q(z)\}>0$ in $\mathcal{U}$. Writing

$$
\frac{F(z)}{z}=p(z) *\left(1+\sum_{m=2}^{\infty} \frac{4(\gamma-\lambda)}{m^{2}[2 \gamma+(\delta-\gamma)(m-1)]} z^{m-1}\right)
$$

and making use of Lemma 3.3 gives that $\operatorname{Re}\left\{\frac{F(z)}{z}\right\}>\frac{1}{2}$ for $z \in \mathcal{U}$.

Lemma 3.5. Let $F_{i} \in \mathcal{R}(\gamma, \delta, \lambda)$ for $i=1,2$. Then $F_{1} * F_{2} \in \mathcal{R}(\gamma, \delta, \lambda)$. 
Proof. Suppose $F_{1}(z)=z+\sum_{m=2}^{\infty} A_{m} z^{m}$ and $F_{2}(z)=z+\sum_{m=2}^{\infty} B_{m} z^{m}$. Then the convolution of $F_{1}(z)$ and $F_{2}(z)$ is defined by

$$
F(z)=\left(F_{1} * F_{2}\right)(z)=z+\sum_{m=2}^{\infty} A_{m} B_{m} z^{m} .
$$

Since $F^{\prime}(z)=F_{1}^{\prime}(z) * \frac{F_{2}(z)}{z}, z F^{\prime \prime}(z)=z F_{1}^{\prime \prime}(z) * \frac{F_{2}(z)}{z}$ and $z F^{\prime \prime \prime}(z)=z F_{1}^{\prime \prime \prime}(z) * \frac{F_{2}(z)}{z}$ then we have

$$
\begin{aligned}
& \frac{2 \gamma F^{\prime}(z)+2 \delta z F^{\prime \prime}(z)+(\delta-\gamma) z^{2} F^{\prime \prime \prime}(z)-2 \lambda}{2(\gamma-\lambda)} \\
= & \left(\frac{2 \gamma F_{1}^{\prime}(z)+2 \delta z F_{1}^{\prime \prime}(z)+(\delta-\gamma) z^{2} F_{1}^{\prime \prime \prime}(z)-2 \lambda}{2(\gamma-\lambda)}\right) * \frac{F_{2}(z)}{z} .
\end{aligned}
$$

Since $F_{1} \in \mathcal{R}(\gamma, \delta, \lambda)$,

$$
\operatorname{Re}\left\{\frac{2 \gamma F_{1}^{\prime}(z)+2 \delta z F_{1}^{\prime \prime}(z)+(\delta-\gamma) z^{2} F_{1}^{\prime \prime \prime}(z)-2 \lambda}{2(\gamma-\lambda)}\right\}>0(z \in \mathcal{U})
$$

and using Lemma 3.4, Re $\left\{\frac{F_{2}(z)}{z}\right\}>\frac{1}{2}$ in U. Now applying Lemma 3.3 to (3.1) yields $\operatorname{Re}\left(\frac{2 \gamma F^{\prime}(z)+2 \delta z F^{\prime \prime}(z)+(\delta-\gamma) z^{2} F^{\prime \prime \prime}(z)-2 \lambda}{2(\gamma-\lambda)}\right)>0$ in U. Thus, $F=F_{1} * F_{2} \in \mathcal{R}(\gamma, \delta, \lambda)$.

Now using Lemma 3.5, we prove that the class $\mathcal{R}_{H}^{0}(\gamma, \delta, \lambda)$ is closed under convolutions of its members. We make use of the techniques and methodology introduced by Dorff [6] for convolution.

Theorem 3.6. Let $\mathfrak{f}_{i} \in \mathcal{R}_{H}^{0}(\gamma, \delta, \lambda)$ for $i=1,2$. Then $\mathfrak{f}_{1} * \mathfrak{f}_{2} \in \mathcal{R}_{H}^{0}(\gamma, \delta, \lambda)$.

Proof. Suppose $\mathfrak{f}_{i}=\mathfrak{s}_{i}+\overline{\mathfrak{t}}_{i} \in \mathcal{R}_{H}^{0}(\gamma, \delta, \lambda)(i=1,2)$. Then the convolution of $\mathfrak{f}_{1}$ and $\mathfrak{f}_{2}$ is defined as $\mathfrak{f}_{1} * \mathfrak{f}_{2}=\mathfrak{s}_{1} * \mathfrak{s}_{2}+\overline{\mathfrak{t}_{1} * \mathfrak{t}_{2}}$. In order to prove that $\mathfrak{f}_{1} * \mathfrak{f}_{2} \in \mathcal{R}_{H}^{0}(\gamma, \delta, \lambda)$, we need to prove that $F_{\epsilon}=\mathfrak{s}_{1} * \mathfrak{s}_{2}+\epsilon\left(\mathfrak{t}_{1} * \mathfrak{t}_{2}\right) \in \mathcal{R}(\gamma, \delta, \lambda)$ for each $\epsilon(|\epsilon|=1)$. By Lemma 3.5, the class $\mathcal{R}(\gamma, \delta, \lambda)$ is closed under convolutions for each $\epsilon(|\epsilon|=1), \mathfrak{s}_{i}+\epsilon \mathfrak{t}_{i} \in \mathcal{R}(\gamma, \delta, \lambda)$ for $i=1,2$. Then both $F_{1}$ and $F_{2}$ given by

$$
F_{1}=\left(\mathfrak{s}_{1}-\mathfrak{t}_{1}\right) *\left(\mathfrak{s}_{2}-\epsilon \mathfrak{t}_{2}\right) \text { and } F_{2}=\left(\mathfrak{s}_{1}+\mathfrak{t}_{1}\right) *\left(\mathfrak{s}_{2}+\epsilon \mathfrak{t}_{2}\right),
$$

belong to $\mathcal{R}(\gamma, \delta, \lambda)$. Since $\mathcal{R}(\gamma, \delta, \lambda)$ is closed under convex combinations, then the function

$$
F_{\epsilon}=\frac{1}{2}\left(F_{1}+F_{2}\right)=\mathfrak{s}_{1} * \mathfrak{s}_{2}+\epsilon\left(\mathfrak{t}_{1} * \mathfrak{t}_{2}\right)
$$

belongs to $\mathcal{R}(\gamma, \delta, \lambda)$. Hence $\mathcal{R}_{H}^{0}(\gamma, \delta, \lambda)$ is closed under convolution.

Now we consider the Hadamard product of a harmonic function with an analytic function which is defined by Goodloe [12] as

$$
\widetilde{f} * \varphi=\mathfrak{s} * \varphi+\overline{\mathfrak{t} * \varphi},
$$

where $\mathfrak{f}=\mathfrak{s}+\overline{\mathfrak{t}}$ is harmonic function and $\varphi$ is an analytic function in $\mathcal{U}$.

Theorem 3.7. Let $\mathfrak{f} \in \mathcal{R}_{H}^{0}(\gamma, \delta, \lambda)$ and $\varphi \in \mathcal{A}$ be such that $\operatorname{Re}\left(\frac{\varphi(z)}{z}\right)>\frac{1}{2}$ for $z \in \mathcal{U}$, then $\mathfrak{f} * \varphi \in \mathcal{R}_{H}^{0}(\gamma, \delta, \lambda)$.

Proof. Suppose that $\mathfrak{f}=\mathfrak{s}+\overline{\mathfrak{t}} \in \mathcal{R}_{H}^{0}(\gamma, \delta, \lambda)$, then $F_{\epsilon}=\mathfrak{s}+\epsilon \mathfrak{t} \in \mathcal{R}(\gamma, \delta, \lambda)$ for each $\epsilon$ $(|\epsilon|=1)$. By Theorem 2.2, in order to show that $\mathfrak{f} \widetilde{*} \varphi \in \mathcal{R}_{H}^{0}(\gamma, \delta, \lambda)$, we need to show that 
$G=\mathfrak{s} * \varphi+\epsilon(\mathfrak{t} * \varphi) \in \mathcal{R}(\gamma, \delta, \lambda)$ for each $\epsilon(|\epsilon|=1)$. Write $G$ as $G=F_{\epsilon} * \varphi$, and

$$
\begin{aligned}
& \frac{1}{2(\gamma-\lambda)}\left(2 \gamma G^{\prime}(z)+2 \delta z G^{\prime \prime}(z)+(\delta-\gamma) z^{2} G^{\prime \prime \prime}(z)-2 \lambda\right) \\
= & \frac{1}{2(\gamma-\lambda)}\left(2 \gamma F_{\epsilon}^{\prime}(z)+2 \delta z F_{\epsilon}^{\prime \prime}(z)+(\delta-\gamma) z^{2} F_{\epsilon}^{\prime \prime \prime}(z)-2 \lambda\right) * \frac{\varphi(z)}{z} .
\end{aligned}
$$

Since $\operatorname{Re}\left(\frac{\varphi(z)}{z}\right)>\frac{1}{2}$ and $\operatorname{Re}\left\{2 \gamma F_{\epsilon}^{\prime}(z)+2 \delta z F_{\epsilon}^{\prime \prime}(z)+(\delta-\gamma) z^{2} F_{\epsilon}^{\prime \prime \prime}(z)-2 \lambda\right\}>0$ in $\mathcal{U}$, Lemma 3.3 proves that $G \in \mathcal{R}(\gamma, \delta, \lambda)$.

Corollary 3.8. Let $\mathfrak{f} \in \mathcal{R}_{H}^{0}(\gamma, \delta, \lambda)$ and $\varphi \in \mathcal{K}$, then $f \widetilde{*} \varphi \in \mathcal{R}_{H}^{0}(\gamma, \delta, \lambda)$.

Proof. Suppose $\varphi \in \mathcal{K}$, then $\operatorname{Re}\left(\frac{\varphi(z)}{z}\right)>\frac{1}{2}$ for $z \in \mathcal{U}$. As a corollary of Theorem 3.7, $f \widetilde{*} \in \in \mathcal{R}_{H}^{0}(\gamma, \delta, \lambda)$.

\section{Radii of fully convexity and starlikeness}

In this section, we obtain the radii of fully convexity and starlikeness of the class $\mathcal{R}_{H}^{0}(\gamma, \delta, \lambda)$. Also, estimates on $\lambda$ that would ensure fully convexity of functions of $\mathcal{R}_{H}^{0}(1, \delta, \lambda)$ are found.

First, we state the following lemmas give sufficient conditions for functions $\mathfrak{f}$ in $\mathcal{H}^{0}$ to belong to $\mathcal{F K}_{H}^{0}$ and $\mathcal{F S}_{H}^{*, 0}$ respectively.

Lemma 4.1 ([21], Corollary 1). Let $\mathfrak{f}=\mathfrak{s}+\overline{\mathfrak{t}}$, where $\mathfrak{s}$ and $\mathfrak{t}$ are given by (1.1). Further, let

$$
\sum_{m=2}^{\infty} m^{2}\left[\left|a_{m}\right|+\left|b_{m}\right|\right] \leq 1
$$

Then $\mathfrak{f}$ is harmonic univalent in $\mathcal{U}$, and $\mathfrak{f} \in \mathcal{F K}_{H}^{0}$.

Lemma $4.2([21]$, Theorem 1$)$. Let $\mathfrak{f}=\mathfrak{s}+\overline{\mathfrak{t}}$, where $\mathfrak{s}$ and $\mathfrak{t}$ are given by (1.1). Further, let

$$
\sum_{m=2}^{\infty} m\left[\left|a_{m}\right|+\left|b_{m}\right|\right] \leq 1
$$

Then $\mathfrak{f}$ is harmonic univalent in $\mathcal{U}$, and $\mathfrak{f} \in \mathcal{F S}_{H}^{*, 0}$.

The following lemma are useful in the proof of the theorems:

Lemma 4.3 ([11,16]). We have

$$
\begin{aligned}
\text { (i) } \sum_{m=2}^{\infty} m r^{m-1} & =\frac{r(2-r)}{(1-r)^{2}}, \\
\text { (ii) } \sum_{m=2}^{\infty} m^{2} r^{m-1} & =\frac{r\left(4-3 r+r^{2}\right)}{(1-r)^{3}} .
\end{aligned}
$$

Theorem 4.4. Let $\mathfrak{f}=\mathfrak{s}+\overline{\mathfrak{t}} \in \mathcal{R}_{H}^{0}(\gamma, \delta, \lambda)$. Then $\mathfrak{f}$ is fully convex in $|z|<r_{c}$, where $r_{c}$ is the unique real root of $p c(r)=0$ in $(0,1)$, and where

$$
p c(r)=(-\delta-2 \gamma+\lambda) r^{3}+(3 \delta+6 \gamma-3 \lambda) r^{2}+(-3 \delta-7 \gamma+4 \lambda) r+\delta+\gamma .
$$

Proof. Let $\mathfrak{f}=\mathfrak{s}+\overline{\mathfrak{t}} \in \mathcal{R}_{H}^{0}(\gamma, \delta, \lambda)$ where $\mathfrak{s}(z)=z+\sum_{m=2}^{\infty} a_{m} z^{m}$ and $\mathfrak{t}(z)=\sum_{m=2}^{\infty} b_{m} z^{m}$. For $r \in(0,1)$, it is sufficient to show that $\mathfrak{f}_{r} \in \mathcal{F J}_{H}^{0}$ where

$$
\mathfrak{f}_{r}(z)=\frac{\mathfrak{f}(r z)}{r}=z+\sum_{m=2}^{\infty} a_{m} r^{m-1} z^{m}+\overline{\sum_{m=2}^{\infty} b_{m} r^{m-1} z^{m}} .
$$


Consider the sum

$$
S=\sum_{m=2}^{\infty} m^{2}\left(\left|a_{m}\right|+\left|b_{m}\right|\right) r^{m-1} .
$$

In view of Theorem 2.7 (i) and (4.4), (4.6) gives

$$
\begin{aligned}
S & \leq \sum_{m=2}^{\infty} m^{2}\left(\frac{4(\gamma-\lambda)}{m^{2}[2 \gamma+(\delta-\gamma)(m-1)]}\right) r^{m-1} \\
& \leq \frac{\gamma-\lambda}{\delta+\gamma} \sum_{m=2}^{\infty} m^{2} r^{m-1} \\
& =\frac{\gamma-\lambda}{\delta+\gamma} \frac{r\left(4-3 r+r^{2}\right)}{(1-r)^{3}}=: X_{1} .
\end{aligned}
$$

Lemma 4.1 implies that in order to show that $\mathfrak{f}_{r} \in \mathcal{F X}_{H}^{0}$, it is sufficient to show that $X_{1} \leq 1$. A simple computation shows that $X_{1} \leq 1$ whenever $p c(r) \geq 0$ where $p c(r)$ is defined by (4.5). It is easy to observe that $p c(0)=\delta+\gamma>0$ and $p c(1)=2(\lambda-\gamma)<0$, and hence $p c(r)$ has at least one root in $(0,1)$.

To show that $p c(r)$ has exactly one root in $(0,1)$, it is sufficient to prove that $p c(r)$ is monotonic function on $(0,1)$. A simple computation shows that

$$
\begin{aligned}
p c^{\prime}(r) & =(-6 \gamma+3 \lambda-3 \delta) r^{2}+(12 \gamma-6 \lambda+6 \delta) r-3 \delta-7 \gamma+4 \lambda \\
p c^{\prime}(0) & =-3 \delta-7 \gamma+4 \lambda=-3(\gamma+\delta)-4(\gamma-\lambda)<0 \\
p c^{\prime}(1) & =\lambda-\gamma<0 \\
p c^{\prime \prime}(r) & =(-6 \delta-12 \gamma+6 \lambda) r+6 \delta+12 \gamma-6 \lambda \\
& =[-6(\gamma+\delta)-6(\delta-\lambda)] r-[-6(\gamma+\delta)-6(\delta-\lambda)] \\
& =[-6(\gamma+\delta)-6(\delta-\lambda)](r-1)>0 \text { for } r \in(0,1) .
\end{aligned}
$$

Hence $p c^{\prime}(r)$ is a strictly monotonic increasing function on $(0,1)$. Since $p c^{\prime}(1)<0$, we conclude that $p c^{\prime}(r)<0$ on $(0,1)$. This shows that $p c(r)$ is strictly monotonically decreasing on $(0,1)$. Thus $p c(r)=0$ has exactly one root in $(0,1)$. Since $p c(r)$ is strictly monotonically decreasing on $(0,1)$ with $p c(0)>0$ and $p c\left(r_{c}\right)=0$, it is easy to see that $p c(r) \geq 0$ for $0<r \leq r_{c}$. Hence $\mathfrak{f}$ is fully convex in $|z|<r_{c}$.

Theorem 4.5. Let $\mathfrak{f}=\mathfrak{s}+\overline{\mathfrak{t}} \in \mathcal{R}_{H}^{0}(\gamma, \delta, \lambda)$. Then $\mathfrak{f}$ is fully starlike in $|z|<r_{s}$, where $r_{s}$ is the unique real root of $p s(r)=0$ in $(0,1)$, and where

$$
p s(r)=(\delta+2 \gamma-\lambda) r^{2}+(-2 \delta-4 \gamma+2 \lambda) r+\delta+\gamma .
$$

Proof. Let $\mathfrak{f}=\mathfrak{s}+\overline{\mathfrak{t}} \in \mathcal{R}_{H}^{0}(\gamma, \delta, \lambda)$. For $r \in(0,1)$, let

$$
\mathfrak{f}_{r}(z)=\frac{\mathfrak{f}(r z)}{r}=z+\sum_{m=2}^{\infty} a_{m} r^{m-1} z^{m}+\overline{\sum_{m=2}^{\infty} b_{m} r^{m-1} z^{m}}
$$

Consider the sum

$$
S=\sum_{m=2}^{\infty} m\left(\left|a_{m}\right|+\left|b_{m}\right|\right) r^{m-1}
$$

Using Theorem 2.7(i) and (4.3), (4.8) gives

$$
\begin{aligned}
S & \leq \sum_{m=2}^{\infty} m\left(\frac{4(\gamma-\lambda)}{m^{2}[2 \gamma+(\delta-\gamma)(m-1)]}\right) r^{m-1} \\
& \leq \frac{\gamma-\lambda}{\delta+\gamma} \sum_{m=2}^{\infty} m r^{m-1} \\
& =\frac{\gamma-\lambda}{\delta+\gamma} \frac{r(2-r)}{(1-r)^{2}}=: X_{2} .
\end{aligned}
$$


In view of Lemma 4.2 in order to prove that $\mathfrak{f}_{r} \in \mathcal{F} \mathcal{S} \mathcal{H}^{*, 0}$, it is sufficient to show that $X_{2} \leq 1$. A simple computation shows that $X_{2} \leq 1$ whenever $p s(r) \geq 0$ where $p s(r)$ is defined by (4.7). It is easy to observe that $p s(0)=\delta+\gamma>0$ and $p s(1)=\lambda-\gamma<0$, and hence $p s(r)$ has a real root in $(0,1)$.

To show that $p s(r)$ has exactly one root in $(0,1)$, it is sufficient to prove that $p s(r)$ is monotonic function on $(0,1)$. A simple computation shows that

$$
\begin{aligned}
p s^{\prime}(r) & =2(\delta+2 \gamma-\lambda)(r-1)<0, \text { for } r \in(0,1) \\
p s^{\prime}(0) & =2(\lambda-\delta-2 \gamma), \\
p s^{\prime}(1) & =0, \\
p s^{\prime \prime}(r) & =2(\delta+2 \gamma-\lambda)>0 .
\end{aligned}
$$

Hence $p s^{\prime}(r)$ is a strictly monotonically increasing function on $(0,1)$. Since $p s^{\prime}(1)=0$, we conclude that $p s^{\prime}(r)<0$ on $(0,1)$. This shows that $p s(r)$ is strictly monotonically decreasing on $(0,1)$. Thus $p s(r)$ has exactly one root in $(0,1)$. Since $p s(r)$ is strictly monotonically decreasing on $(0,1)$ with $p s(0)>0$ and $p s\left(r_{s}\right)=0$, it is easy to see that $p s(r) \geq 0$ for $0<r \leq r_{s}$. Hence $\mathfrak{f}$ is fully starlike in $|z|<r_{s}$.

Lemma 4.6 ([2], Corollary 3.2). Let $\lambda<1, \delta \geq 1$, and $\mathfrak{f} \in \mathcal{R}(1, \delta, \lambda)$. If $\lambda$ satisfies

$$
7-3 \delta=4 \lambda+4(1-\lambda) \sum_{m=1}^{\infty} \frac{2 m(3-\delta)+(\delta-5)}{(m+1)(m(\delta-1)+2)},
$$

then $\mathfrak{f}$ is convex in $\mathcal{U}$.

Theorem 4.7. Suppose $\mathfrak{f} \in \mathcal{R}_{H}^{0}(1, \delta, \lambda)$ with $\lambda<1, \delta \geq 1$. If $\lambda$ satisfies (4.9), then $\mathfrak{f}$ is fully convex in U.

Proof. Let $\lambda<1, \delta \geq 1$ and $\mathfrak{f} \in \mathcal{R}_{H}^{0}(1, \delta, \lambda)$. Then $F_{\epsilon}=\mathfrak{s}+\epsilon \mathfrak{t} \in \mathcal{R}(1, \delta, \lambda)$ for each $\epsilon(|\epsilon|=1)$. If $\lambda$ satisfies (4.9), then $F_{\epsilon}$ is convex in U. In view of [17, Corollary 2.4], it follows that $\mathfrak{f}$ is fully convex in $\mathcal{U}$.

In [2, Example 3.1], it is stated that if $\mathfrak{f} \in \mathcal{R}(1,3, \lambda)$ then $\mathfrak{f}$ is convex in $\mathcal{U}$ whenever $\lambda$ satisfies $\lambda=\frac{\pi^{2}-9}{\pi} \approx 0.088843$. However [1, Theorem 5.3] through the use of techniques in duality, obtained the sharp estimate $\lambda=\frac{1-\ln 2}{2(1-\ln 2)} \equiv-0,629445$. Thus, Rosihan et al. [2] motivated the following problem:

Problem 4.8 ([2], Problem 3.1). Find $\gamma, \delta$ and $\lambda$ so that functions $\mathfrak{f} \in \mathcal{R}(\gamma, \delta, \lambda)$ are convex in $\mathcal{U}$.

Now, we extend this problem to

Problem 4.9. Find $\gamma, \delta$ and $\lambda$ so that functions $\mathfrak{f} \in \mathcal{R}_{H}^{0}(\gamma, \delta, \lambda)$ are fully convex in $\mathcal{U}$.

\section{References}

[1] R.M. Ali, M.M. Nargesi and V. Ravichandran, Convexity of integral transforms and duality, Complex Var. Elliptic Equ. 58 (11), 1569-1590, 2013.

[2] R.M. Ali, D. Satwanti and A. Swaminathan, Inclusion properties for a class of analytic functions defined by a second-order differential inequality, RACSAM, 112, 117-133, 2018.

[3] P.N. Chichra, New subclasses of the class of close-to-convex functions, Proc. Am. Math. Soc. 62 (1), 37-43, 1976.

[4] M. Chuaqui, P. Duren and B. Osgood, Curvature properties of planar harmonic mappings, Comput. Methods Funct. Theory, 4 (1), 127-142, 2004.

[5] J. Clunie and T. Sheil-Small, Harmonic univalent functions, Ann. Acad. Sci. Fenn. Ser. A I 9, 3-25, 1984. 
[6] M. Dorff, Convolutions of planar harmonic convex mappings, Complex Var. Theory Appl., 45 (3), 263-271, 2001.

[7] P. Duren, Univalent Functions, in: Grundlehren Der Mathematischen Wissenschaften, vol. 259, Springer-Verlag, New York, Berlin, Heidelberg, Tokyo, 1983.

[8] P. Duren, Harmonic mappings in the plane, Cambridge Tracts in Mathematics, 156, Cambridge Univ. Press, Cambridge, 2004.

[9] L. Fejér, Über die Positivität von Summen, die nach trigonometrischen oder Legendreschen Funktionen fortschreiten, Acta Litt. Ac Sei. Szeged, 75-86, 1925.

[10] N. Ghosh and A. Vasudevarao, On a subclass of harmonic close-to-convex mappings, Monatsh. Math., 188, 247-267, 2019.

[11] N. Ghosh and A. Vasudevarao, The radii of fully starlikeness and fully convexity of a harmonic operator, Monatsh Math., 188, 653-666, 2019.

[12] M. Goodloe, Hadamard products of convex harmonic mappings, Complex Var. Theory Appl., 47 (2), 81-92, 2002.

[13] R. Herandez and M.J. Martin, Stable geometric properties of analytic and harmonic functions, Math. Proc. Cambridge Philos. Soc. 155, 343-359, 2013.

[14] S.S. Miller and P.T. Mocanu, Differential subordinations and univalent functions, Michigan Math. J. 28 (2), 157-171, 1981.

[15] S.S. Miller and P.T Mocanu, Differential Subordinations, Theory and Applications, Marcel Dekker, New York, Basel, 1999.

[16] S. Nagpal and V. Ravichandran, Fully starlike and fully convex harmonic mappings of order $\alpha$, Ann. Polon. Math. 108 (1), 85-107, 2013.

[17] S. Nagpal and V. Ravichandran, Construction of subclasses of univalent harmonic mappings, J. Korean Math. Soc., 53, 567-592, 2014.

[18] Rajbala, J.K. Prajapat, On a subclass of close-to-convex harmonic mappings, AsianEuropean Jour Math., 14 (06), 2150102, 2021.

[19] O. Al-Refai, Some properties for a class of analytic functions defined by a higher-order differential inequality, Turkish J. Math., 43, 2473-2493, 2019.

[20] R.M. Ali, S.K. Lee, K.G. Subramanian and A. Swaminathan, A third order differential equation and starlikeness of a double integral operator, Abst. Appl. Anal., Article ID 901235, 2011.

[21] H, Silverman, Harmonic univalent functions with negative coefficients, Jour. Math. Anal. Appl., 220, 283-289, 1998.

[22] R. Singh and S. Singh, Convolution properties of a class of starlike functions, Proc. Amer. Math. Soc., 106, 145-152, 1989.

[23] E. Yaşar and S. Yalçın, Close-to-convexity of a class of harmonic mappings defined by a third-order differential inequality, Turkish J. Math., 45 (2), 678-694, 2021. 